\title{
Initial surgical experience following implementation of lung cancer screening at an urban safety net hospital
}

Juan A. Muñoz-Largacha, MD, ${ }^{\mathrm{a}}$ Katrina A. Steiling, MD, ${ }^{\mathrm{b}}$ Hasmeena Kathuria, MD, ${ }^{\mathrm{b}}$ Marjory Charlot, MD, MPH, ${ }^{\mathrm{c}}$ Carmel Fitzgerald, RN, ${ }^{\mathrm{b}}$ Kei Suzuki, MD, ${ }^{\mathrm{a}}$ and Virginia R. Litle, MD ${ }^{\mathrm{a}}$

\section{ABSTRACT}

Background: Safety net hospitals provide care mostly to low-income, uninsured, and vulnerable populations, in whom delays in cancer screening are established barriers. Socioeconomic barriers might pose important challenges to the success of a lung cancer screening program at a safety net hospital. We aimed to determine screening follow-up compliance, rates of diagnostic and treatment procedures, and the rate of cancer diagnosis in patients classified as category 4 by the Lung CT Screening Reporting and Data System (Lung-RADS 4).

Methods: We conducted a retrospective review of all patients enrolled in our multidisciplinary lung cancer screening program between March 2015 and July 2016. Demographics, smoking status, Lung-RADS score, and number of diagnostic and therapeutic interventions and cancer diagnoses were captured.

Results: A total of 554 patients were screened over a 16-month period. The mean patient age was 63 years (range, $47-85$ years), and $60 \%$ were male. The majority $(92 \% ; 512$ of 554) were classified as Lung-RADS 1 to 3 , and $8 \%$ (42 of 554) were classified as Lung-RADS 4. Among the Lung-RADS 4 patients, 98\% (41 of 42) completed their recommended follow-up; 29\% (12 of 42) underwent a diagnostic procedure, for an overall diagnostic intervention rate of $2 \%$ (12 of 554). Eleven of these 12 patients had cancer, and 1 patient had sarcoidosis. The overall rate of surgical resection was $0.9 \%$ ( 5 of 554), and the rate of diagnostic intervention for noncancer diagnosis was $0.1 \%$ (1 of 554).

Conclusions: Implementation of a multidisciplinary lung cancer screening program at a safety net hospital is feasible. Compliance with follow-up and interventional recommendations in Lung-RADS 4 patients was high despite anticipated social challenges. Overall diagnostic and surgical resection rates and interventions for noncancer diagnosis were low in our initial experience. ( $\mathrm{J}$ Thorac Cardiovasc Surg 2018;155:2674-81)

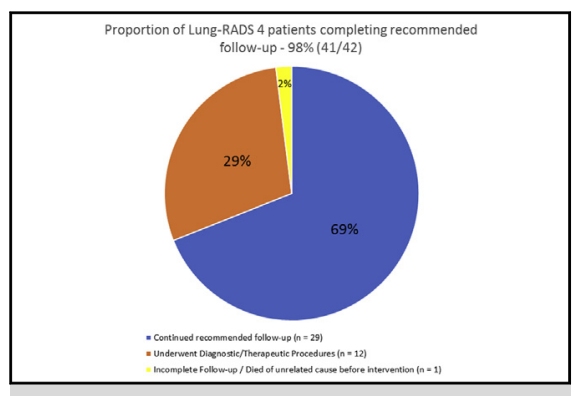

The proportion of Lung-RADS 4 patients completing recommended follow-up was $98 \%$ (41 of 42 ).

Central Message
Implementation of a multidisciplinary lung
cancer screening program at a safety net hospi-
tal resulted in high compliance with follow-up
in low income, minority patients at elevated
risk of poor outcomes.

\section{Perspective}

Safety net hospitals provide care to lowincome, uninsured populations in whom delays in cancer screening are established barriers. Implementing a multidisciplinary lung cancer screening program at a safety net hospital results in high follow-up compliance and interventional recommendations in patients classified as Lung-RADS 4, with low rates of surgical resection and interventions for noncancer diagnoses.

See Editorial Commentary page 2682.

\footnotetext{
From the ${ }^{\mathrm{a} D i v i s i o n}$ of Thoracic Surgery, Department of Surgery, ${ }^{\mathrm{b}}$ Division of Pulmonary, Allergy, Sleep and Critical Care Medicine, Department of Medicine, and ${ }^{\mathrm{c}} \mathrm{Di}$ vision of Hematology and Medical Oncology, Department of Medicine, Boston University School of Medicine, Boston, Mass.

Drs Muñoz-Largacha and Steiling contributed equally to this work.

Read at the 43rd Annual Meeting of The Western Thoracic Surgical Association, Colorado Springs, Colorado, June 21-24, 2017.

Received for publication June 19, 2017; revisions received Dec 14, 2017; accepted for publication Dec 14, 2017; available ahead of print March 11, 2018.

Address for reprints: Virginia R. Litle, MD, 88 East Newton St, Collamore C-7380, Boston, MA 02118 (E-mail: Virginia.litle@bmc.org).

0022-5223/\$36.00

Copyright (c) 2018 by The American Association for Thoracic Surgery

https://doi.org/10.1016/j.jtcvs.2017.12.135
}

Lung cancer screening with low-dose computed tomography (LDCT) was associated with reduced lung cancer mortality in a high-risk population of smokers in the National Lung Screening Trial (NLST). ${ }^{1}$ These results have increased the general awareness of the importance and utility of lung cancer screening and spurred the creation of new

Scanning this QR code will take you to the article title page.

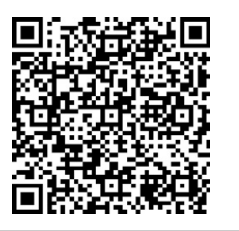




\begin{tabular}{|c|c|}
\hline \multicolumn{2}{|c|}{ Abbreviations and Acronyms } \\
\hline CT & $=$ computed tomography \\
\hline $\mathrm{FEV}_{1}$ & $\begin{aligned}= & \text { forced expiratory volume in } \\
& 1 \text { second }\end{aligned}$ \\
\hline LDCT & $=$ low-dose computed tomography \\
\hline Lung-RAD & $\begin{aligned} \mathrm{S}= & \text { Lung CT Screening Reporting and } \\
& \text { Data System }\end{aligned}$ \\
\hline MDT & $=$ multidisciplinary team \\
\hline NLST & $=$ National Lung Screening Trial \\
\hline USPSTF & $\begin{aligned}= & \text { United States Preventive Services } \\
& \text { Task Force }\end{aligned}$ \\
\hline VATS & $=$ video-assisted thoracoscopic surgery \\
\hline
\end{tabular}

guidelines and recommendations from such organizations as the National Comprehensive Cancer Network in 2012, the American Cancer Society in 2013, and the US Preventive Services Task Force (USPSTF) in 2014, among others. ${ }^{2-5}$

Regardless of the guidelines used for lung cancer screening, it has been well established that lung cancer screening should be performed in high-volume, high-quality (ie, designated), and experienced centers with expertise in LDCT screening. ${ }^{5,6}$ Moreover, the adoption of a standardized Lung CT Screening Reporting and Data System (Lung-RADS) and the use of a multidisciplinary team (MDT) comprising pulmonary medicine, thoracic surgery, thoracic radiology, radiation oncology, medical oncology, and pathology are additional factors that have proven essential for lung cancer screening success. ${ }^{5-8}$ The Lung-RADS is an essential tool that was first proposed in 2014 to facilitate communication among clinicians involved in the screening process and to classify lung nodules according to the risk of malignancy. ${ }^{9}$ Subsequently, the American College of Radiology created an updated version to decrease the false-positive results after lung cancer screening. ${ }^{10}$ This classification system categorizes patients into 1 of 4 assessment categories according to the risk of malignancy, with a probability of malignancy of $<1 \%$ for patients classified as Lung-RADS 1-2 and a risk of $2 \%$ for those classified as Lung-RADS 3. The risk of malignancy increases for those categorized as Lung-RADS category $4 \mathrm{~A}(5 \%-15 \%)$ and for those categorized as Lung-RADS categories $4 \mathrm{~B}$ and $4 \mathrm{X}$ (risk of malignancy $>15 \%)^{11}$

Safety net hospitals are defined as organizations that provide and deliver most of their care to low-income, uninsured, and vulnerable populations. ${ }^{12}$ These populations frequently have higher rates of smoking, and delays in cancer screening and diagnosis are established barriers. ${ }^{13-16}$ Thus, we hypothesized that these socioeconomic barriers might pose important challenges for the implementation and success of a lung cancer screening program at an urban safety net hospital like Boston Medical Center.
Moreover, these barriers may impact guideline the concordant follow-up and management of patients at risk for poor outcomes, such as those classified as LungRADS 4. Therefore, in the present study, we aimed to determine the rates of LDCT follow-up compliance, diagnostic and treatment procedures, and cancer diagnosis in LungRADS 4 patients.

\section{METHODS}

Institutional Review Board approval was granted for this study (IRB ID \#H-35216). We conducted a retrospective review of all patients enrolled in our MDT lung cancer screening program between March 2015 (the start date for the lung cancer screening program at our institution) and July 2016. Demographic information (age, sex, race/ethnicity), smoking history, insurance status, radiologic findings, Lung-RADS category, the number of diagnostic and therapeutic interventions, and cancer diagnoses were captured for the 554 patients screened during the study period. The USPSTF criteria were used for screening eligibility. Most patients met the age criteria (55-80 years), and just a small proportion were younger than 55 or older than 80 years but were screened based on the ordering physician's clinical judgment based on other risk factors (eg, strong smoking history, lung nodule follow-up, family history of lung cancer). In most cases, the referral for lung cancer screening was initiated by a primary care provider, internist, or pulmonologist. In all cases, the Lung-RADS was used to classify the patients after LDCT into 1 of the 4 defined categories. Data from patients who had initial LDCT results suspicious for malignancy and were categorized as Lung-RADS 4 were used for analysis. All Lung-RADS 4 patients were evaluated by a MDT comprising nurse navigators, primary care physicians, pulmonologists, thoracic surgeons, thoracic radiologists, medical oncologists, and pathologists. Captured variables included diagnostic interventions, surgical procedures, postoperative diagnosis, histological and pathological staging, as well as follow-up compliance with screening recommendations.

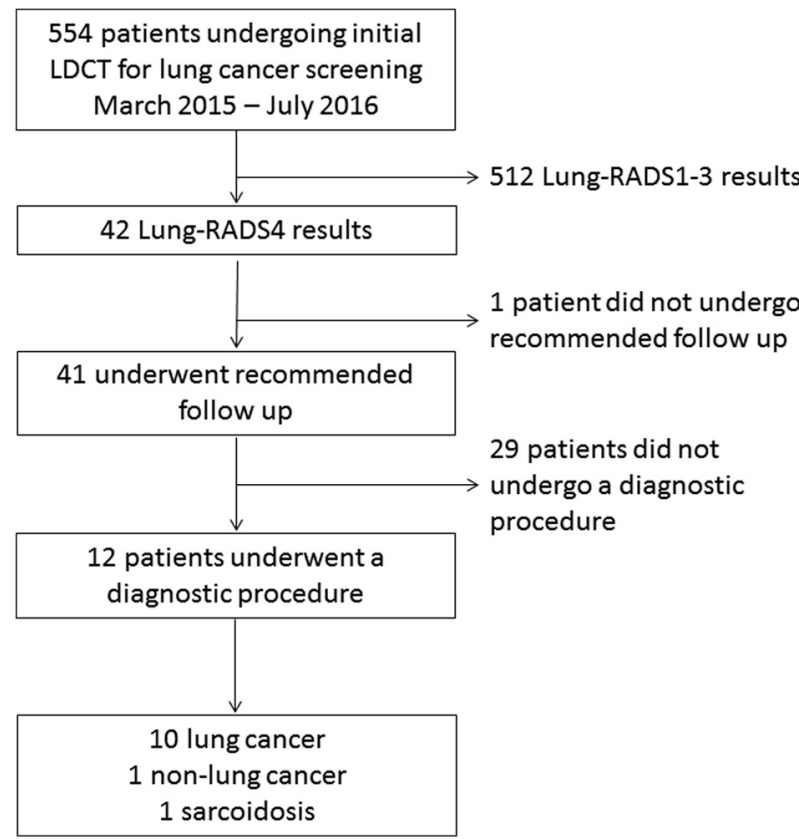

FIGURE 1. Flow diagram showing the results of our retrospective analysis. 


\section{RESULTS}

A total of 554 patients were screened in our MDT program between March 6, 2015, and July 29, 2016 (Figure 1). The mean patient age was 63 years (range, 4785 years), with $95 \%$ meeting the USPSTF eligibility age criteria (55-80 years). Sixty percent were male. The racial/ethnic distribution was $48 \%$ non-Hispanic white, $31 \%$ non-Hispanic Black, $10 \%$ Hispanic, and 5\% Asian and Native American; in $6 \%$, this information was not documented.

All patients had a history of cigarette smoking. Slightly more than one-half $(52 \% ; 286$ of 554) were current smokers, $25 \%$ (136 of 554) were former smokers, and in $23 \%$ (132 of 554), information on active smoking was not available (Table 1). Eighty-one percent (447/554) met the USPSTF criterion of smoking more than 30 packyears, 7 patients had a $<30$ pack-year history, and this information was not available in the remaining 18\% (100 of 554). Information on smoking quit date was available in $22 \%$ (121 of 554 ), of which $97 \%$ (117 of 121 ) met the USPSTF eligibility criterion of quitting within the past 15 years.

Almost all patients were asymptomatic, with the exception of 15 patients with weight loss and 2 patients with hemoptysis. However, among the 17 patients with weight loss or hemoptysis, just 2 patients had suspicious findings after screening LDCT. Sixty-four percent of the patients were covered by Medicare or Medicaid, and the remaining $36 \%$ had private insurance. Screening referral came from primary care physicians in $62 \%$ of the patients, from pulmonary/medicine services in $21 \%$, and from other services, such as thoracic surgery, other inpatient and outpatient providers, and emergency room physicians, in the remaining $17 \%$.

The initial LDCT screening exams of 92\% (512 of 554) of the patients were categorized as Lung-RADS 1 to 3, and the remaining $8 \%$ (42 of 554) were categorized as LungRADS 4 . The 42 Lung-RADS 4 patients included 27 males (64\%), and the mean age was 64 years (range, 54-81 years). Fifty-seven percent were current smokers with a mean number of pack-years of 44.5 (range 30-100), 26\% were former smokers (mean pack-years, 45.1 ; range, $30-90$ years), and in $17 \%$ of the patients, this information was not documented. Fifty-seven percent were non-Hispanic white, $24 \%$ were African-American, 7\% were Hispanic, 10\% were Asian, and 1 patient declined to provide this information. Sixtynine percent had Medicare/Medicaid, and the remaining $31 \%$ had private insurance. All patients were asymptomatic, except for 1 patient who experienced hemoptysis and was later diagnosed with inoperable stage IIIA non-small cell lung cancer and another patient who reported weight loss and was subsequently diagnosed with gastric cancer. In this group, $98 \%$ (41 of 42 ) of the patients completed their
TABLE 1. Clinical characteristics

\begin{tabular}{lc}
\hline \multicolumn{1}{c}{ Variable } & Number (\%) \\
\hline Total number of patients & $554(100)$ \\
Sex & \\
$\quad$ Female & $221(40)$ \\
Male & $333(60)$ \\
Smoking status & \\
Former & $136(25)$ \\
Current & $286(52)$ \\
Undetermined & $132(23)$ \\
Race/ethnicity & \\
Non-Hispanic white & $265(48)$ \\
Non-Hispanic black & $174(31)$ \\
Hispanic & $56(10)$ \\
Asian/Native American & $28(5)$ \\
Declined/not available & $31(6)$ \\
\hline
\end{tabular}

recommended follow-up according to the Lung-RADS management guidelines, and 1 patient died from an unrelated cause before diagnostic intervention.

Twelve of the $42(29 \%)$ Lung-RADS 4 patients underwent a diagnostic procedure, for an overall diagnostic intervention rate of $2 \%$ (12 of 554). Three patients underwent computed tomography (CT)-guided biopsy, 3 patients underwent bronchoscopy and mediastinoscopy, 1 patient underwent bronchoscopy alone, 3 patients underwent diagnostic video-assisted thoracoscopic surgery (VATS), 1 patient underwent electromagnetic navigational bronchoscopy and mediastinoscopy, and 1 patient had an ultrasound (US)-guided biopsy from a liver lesion that proved to be a metastatic lesion from small cell lung cancer (Table 2). The mean interval between the screening CT scan and the initial diagnostic procedure was $52 \pm 23$ days (range, 2196 days). The mean interval between the screening CT scan and first positive cytology or pathology specimen was $55 \pm 26$ days (range, 21-96 days). All 12 patients underwent a positron emission tomography (PET) scan. The mean interval between the initial screening CT and the PET scan was $30 \pm 12$ days (range, 9-55 days). Of the 12

TABLE 2. Diagnostic procedures performed in the Lung-RADS 4 patients $(\mathbf{n}=12)$

\begin{tabular}{lc}
\hline \multicolumn{1}{c}{ Diagnostic procedure } & Number $(\%)$ \\
\hline Computed tomography-guided biopsy & $3(25)$ \\
Bronchoscopy and mediastinoscopy & $3(25)$ \\
Bronchoscopy & $1(8)$ \\
Diagnostic VATS & $3(25)$ \\
ENB and mediastinoscopy & $1(8)$ \\
Ultrasound-guided biopsy & $1(8)$ \\
\hline
\end{tabular}

VATS, Video-assisted thoracoscopic surgery; $E N B$, electromagnetic navigational bronchoscopy. 
TABLE 3. Pathology results after diagnostic procedures $(n=12)$

\begin{tabular}{lc}
\hline \multicolumn{1}{c}{ Histology } & Number $(\%)$ \\
\hline Adenocarcinoma & $7(58)$ \\
Squamous cell carcinoma & $2(16)$ \\
Small cell carcinoma & $2(16)$ \\
Sarcoidosis & $1(8)$ \\
\hline
\end{tabular}

Lung-RADS 4 patients who underwent a diagnostic procedure, $10(83 \%)$ completed pulmonary function testing. In these 10 patients, the mean forced expiratory volume in 1 second $\left(\mathrm{FEV}_{1}\right)$ was $1.85 \pm 0.70 \mathrm{~L}\left(\mathrm{FEV}_{1}\right.$ as a percent of predicted, $68 \% \pm 19 \%$ ), and mean CO diffusion capacity of the lung was $11.34 \pm 5.67 \mathrm{~mL} / \mathrm{mm} \mathrm{Hg} / \mathrm{min}$. Of these 12 patients, $11(92 \%)$ were diagnosed with lung cancer (cancer diagnosis rate of $1.9 \% ; 11$ of 554 ), and 1 patient was diagnosed with sarcoidosis (Table 3). Of the 11 patients with lung cancer, $6(55 \%)$ were diagnosed at an early stage (stage IA-IIA), 3 had stage III disease, 1 had stage IV disease, and 1 had limited-stage small cell lung cancer (Table 4). After the diagnostic procedures, 5 patients underwent VATS resection of lung lesions, all with early-stage disease (IA-IIA). Of the 5 patients undergoing resection, 1 patient underwent a VATS wedge resection owing to poor preoperative pulmonary function tests, 2 patients underwent VATS segmentectomy, and 2 patients underwent VATS lobectomy. One patient (with stage IA disease) underwent stereotactic body radiation therapy due to poor pulmonary function tests, 5 patients underwent definitive chemoradiation therapy or chemotherapy alone due to advanced disease (stage IIIA-IV), and the patient with a diagnosis of sarcoidosis continued follow-up with the sarcoidosis clinic (Table 5). The overall surgical resection rate was $0.9 \%$ (5 of $554)$, and the overall intervention rate for noncancer diagnosis was $0.1 \%$ ( 1 of 554). No complications were reported in these 12 patients after any diagnostic and/or therapeutic procedure, and there were no deaths after surgical resection. The interval between the screening CT scan and the initiation of definitive therapy for the 11 patients diagnosed with lung cancer was $85 \pm 37$ days (range, 28-153 days).

TABLE 4. Stages of lung cancer after diagnostic procedures $(n=11)$

\begin{tabular}{lc}
\hline Lung cancer stage & Number $(\%)$ \\
\hline Early stage & $3(27)$ \\
IA & $2(18)$ \\
IB & $1(9)$ \\
IIA & \\
Advanced stage & $2(18)$ \\
IIIA & $1(9)$ \\
IIIB & $1(9)$ \\
IV & $1(9)$ \\
\hline Limited stage*
\end{tabular}

*One patient with small cell lung cancer.
Of the remaining 29 patients whose initial screening scans were classified as Lung-RADS 4 and did not have any invasive diagnostic or therapeutic procedures, $100 \%$ completed Lung-RADS screening recommendations and follow-up. Three-month follow-up LDCT and/or PET scans were performed in all patients. All patients continued recommended follow-up according to new results and established guidelines. One of these patients was later diagnosed with gastric cancer due to new findings on follow-up PET and was treated accordingly, and another patient continued recommended follow-up in a different institution.

\section{DISCUSSION}

Urban safety net hospitals like Boston Medical Center provide care mostly to uninsured and vulnerable populations, characterized by low incomes and high rates of smoking. It is well known that delays in cancer screening and diagnosis are established barriers in this population. ${ }^{13-16}$ Sabatino and colleagues ${ }^{14}$ have shown large screening disparities when analyzing data on breast, cervical, and colorectal cancer screening among individuals of lower socioeconomic status, in those with a lower educational level, and among the Hispanic population. Other factors previously identified as barriers for cancer screening exams include pain and embarrassment associated with screening, poor knowledge of the benefits of screening, lack of physician recommendations, lack of trust with the health system and health personnel, language barriers, and lack of transportation. ${ }^{17,18}$ These observations highlight the importance of addressing barriers to screening when implementing comprehensive cancer screening programs, including those for lung cancer screening, to ensure access for all patients regardless of socioeconomic status, education, and race/ethnicity.

Based on a 2016 Boston Medical Center community needs assessment, we considered these factors when implementing the lung cancer screening program in our center, which serves a traditionally underserved population, including low-income patients, the homeless population, and patients for whom English is a second language. The creation and combined efforts of our MDT and the adoption of the Lung-RADS for a standardized CT scan reporting and classification system have ensured access to our program. Although the program encourages screening of patients meeting the USPSTF eligibility criteria, an initial shortcoming of our program is that some non-USPSTF patients were screened (undocumented pack-year smoking history, quitting date), most of them in the first months of the program, as we allowed ordering clinicians to obtain the exam based on their clinical judgment and their personalized risk/benefit discussion with the patients. However, to avoid screening in ineligible patients, and to make our eligibility process more refined, we have reached out these 
TABLE 5. Therapeutic approaches after cancer diagnosis $(n=11)$

\begin{tabular}{ll}
\hline \multicolumn{1}{c}{ Treatment after diagnosis } & Number $(\%)$ \\
\hline Video-assisted thoracoscopic surgery resection & \\
Wedge* & $1(9)$ \\
Segmentectomy & $2(18)$ \\
Lobectomy & $2(18)$ \\
Stereotactic body radiation therapy & $1(9)$ \\
Definitive chemotherapy/radiotherapy & $3(27)$ \\
Chemotherapy alone & $2(18)$ \\
\hline
\end{tabular}

* One patient had a wedge resection instead of an anatomic resection owing to poor preoperative pulmonary function tests.

providers to educate them and remind them of the USPSTF screening guidelines.

In this study, we examined the proportion of screened patients who were at higher risk of lung cancer based on suspicious findings on screening LDCT, and on whether the patient underwent the recommended follow-up. Our proportion of Lung-RADS 4 patients $(8 \%)$ was higher compared with that in the reanalysis of the NLST $(6.2 \%)^{19}$ and that reported by Walker and colleagues $(6.3 \%) .{ }^{20}$ In contrast, $16.6 \%$ of the patients in an analysis of lung cancer screening performed at the Veterans Health Administration (VHA) had nodules measuring $\geq 8 \mathrm{~mm}^{21}$ In the VHA study, the overall rate of lung cancer among all individuals screened was $1.5 \%,{ }^{21}$ which is slightly lower than the rate of $1.8 \%$ observed in our patient population. In contrast, the rate of lung cancer diagnosed following an initial screening CT exam is higher than that found at centers that screened patients with lower cumulative packyears of smoking. ${ }^{22}$ The differences in the rates of LungRADS 4 findings and lung cancer diagnoses might be explained in part by the inherent social disparities of our patient population associated with higher smoking rates. ${ }^{13}$ Within the NLST, for example, current smokers, those with less than a high school education, and blacks had higher rates of lung cancer death. ${ }^{23}$ Importantly, even though our series comprised mostly socioeconomically disadvantaged patients, compliance with screening and follow-up recommendations was high in our Lung-RADS 4 patients, with $100 \%$ completion of recommended follow-up. This high rate of diagnostic resolution for Lung-RADS 4 exams may be due to our program's use of a dedicated nurse practitioner program coordinator who directly contacts the ordering provider to communicate Lung-RADS 4 results, as well as a patient navigator who helps schedule follow-up exams and referrals for patients with Lung-RADS 4 results.

One critical aspect of lung cancer screening is the potential for harms associated with an increased rate of unnecessary invasive procedures, radiation exposure, psychological distress, and overdiagnosis. ${ }^{6}$ However, our overall diagnostic intervention rate of $2 \%$ was lower than those reported in the literature. ${ }^{1,15,25}$ Moreover, our surgical resection rate $(0.9 \% ; 5$ of 554$)$ and diagnostic intervention rate for noncancer diagnosis $(0.1 \%$; 1 of $554)$ were also lower compared to the NLST data $( \pm 5 \%$ surgical resection rate) ${ }^{1}$ and several previous studies demonstrating surgical resection rates of $1.5 \%$ to $3.6 \%$ with a $0.17 \%$ to $0.6 \%$ intervention rate for noncancer diagnoses. ${ }^{15,22-25}$ This highlights the favorable results of our initial experience after implementation of a lung cancer screening program in a safety net hospital.

We believe that one of the factors that defines screening success is the capability to diagnose these patients at an early stage of the disease. Here we have shown that $55 \%$ of the patients diagnosed with lung cancer were identified at an early stage, results that are also comparable with the NLST data, in which $57 \%$ of patients with lung cancer were diagnosed at an early stage.

Although most patients enrolled in our screening program met the USPSTF eligibility criteria, some patients underwent screening without meeting eligibility criteria due to clinician preferences and risk factors outside of those defined by the USPSTF. We have since implemented programs to improve clinician education on USPSTF guidelines and accuracy of complete data collection on smoking history (eg, pack-years, quit date), with the goal of improving patient selection and program success.

\section{CONCLUSIONS}

Implementing a lung cancer screening program at an urban safety net hospital like Boston Medical Center is feasible. The use of a standardized imaging reporting system (Lung-RADS) and an MDT ensures guidelineconcordant follow-up in high-risk Lung-RADS 4 patients. The compliance with the follow-up and interventional recommendations in our Lung-RADS 4 patients was high despite the challenges inherent to vulnerable populations. Our overall diagnostic and surgical resection rates and intervention rates for noncancer diagnoses were low compared with previously reported data. Specific strategies have been suggested for successfully implementing lung cancer screening, including the use of standardized operating protocols for determining eligibility, screening, results reporting, and results communication. ${ }^{7,25}$ We also believe that our program's use of a nurse practitioner program coordinator and patient navigator are essential components for ensuring high follow-up rates for LungRADS 4 exams in our vulnerable patient population. This highlights our initial success with patient selection, use of Lung-RADS, low invasiveness, and low rate of unnecessary procedures. As in other programs,${ }^{20,21}$ managing workload to maintain the screening registry and ensure timely followup of abnormal screening exams is a potential barrier. Continued efforts are in place to improve our data collection and further improve patient selection and screening. 
The continued growth and improvement of screening programs in large safety net hospitals are essential to improving lung cancer outcomes in this patient population at the greatest risk for lung cancer mortality.

\section{Conflict of Interest Statement}

Authors have nothing to disclose with regard to commercial support.

We thank Nirupama Vellanki, BA for her help with data collection during the initial stages of this project.

\section{References}

1. Aberle DR, Adams AM, Berg CD, Black WC, Clapp JD, Fagerstrom RM, et al; National Lung Screening Trial Research Team. Reduced lung-cancer mortality with low-dose computed tomographic screening. N Engl J Med. 2011;365: 395-409.

2. Wood DE, Eapen GA, Ettinger DS, Hou L, Jackman D, Kazerooni E, et al. Lung cancer screening. J Natl Compr Canc Netw. 2012;10:240-65.

3. Wender R, Fontham ET, Barrera E Jr, Colditz GA, Church TR, Ettinger DS, et al. American Cancer Society lung cancer screening guidelines. CA Cancer J Clin. 2013;63:107-17.

4. Moyer VA. Screening for lung cancer: U.S. preventive services task force recommendation statement. Ann Intern Med. 2014;160:330-8.

5. Smith RA, Manassaram-Baptiste D, Brooks D, Doroshenk M, Fedewa S, Saslow D, et al. Cancer screening in the United States, 2015: a review of current American Cancer Society guidelines and current issues in cancer screening. $C A$ Cancer J Clin. 2015;65:30-54.

6. Tanoue LT, Tanner NT, Gould MK, Silvestri GA. Lung cancer screening. Am J Respir Crit Care Med. 2015;191:19-33.

7. Mazzone P, Powell CA, Arenberg D, Bach P, Detterbeck F, Gould MK, et al. Components necessary for high-quality lung cancer screening: American College of Chest Physicians and American Thoracic Society Policy Statement. Chest. 2015; 147:295-303.

8. Walker BL, Williamson C, Regis SM, McKee AB, D'Agostino RS, Hesketh PJ, et al. Surgical outcomes in a large, clinical, low-dose computed tomographic lung cancer screening program. Ann Thorac Surg. 2015;100:1218-23.

9. Manos D, Seely JM, Taylor J, Borgaonkar J, Roberts HC, Mayo JR. The lung reporting and data system (LU-RADS): a proposal for computed tomography screening. Can Assoc Radiol J. 2014;65:121-34.

10. McKee BJ, Regis SM, McKee AB, Flacke S, Wald C. Performance of ACR LungRADS in a clinical CT lung screening program. J Am Coll Radiol. 2015;12: 273-6.

11. American College of Radiology. Lung-RADS version 1.0 assessment categories release date: April 28, 2014. Available at: https://www.acr.org/-/media/ACR/ Files/RADS/Lung-RADS/LungRADS_AssessmentCategories.pdf. Accessed June 5, 2018.

12. Lewin ME, Altman S, eds. Institute of Medicine (US) Committee on the Changing Market, Managed Care, and the Future Viability of Safety Net Providers. America's Health Care Safety Net. Washington, DC: National Academies Press; 2000 .

13. Levinson AH. Where the US tobacco epidemic still rages: most remaining smokers have lower socioeconomic status. J Health Care Poor Underserved. 2017:28:100-7.

14. Sabatino SA, White MC, Thompson TD, Klabunde CN. Cancer screening test use-United States, 2013. MMWR Morb Mortal Wkly Rep. 2015;64:464-8.

15. White A, Thompson TD, White MC, Sabatino SA, de Moor J, Doria-Rose PV, et al. Cancer screening test use-United States, 2015. MMWR Morb Mortal Wkly Rep. 2017;66:201-6.

16. McCall BM, Wilshire CL, Modin HE, Fathi JT, Gilbert CR, Louie B, et al. MINI01.10: Diverse populations may be underrepresented in community lung cancer screening programs: topic: pulmonology. J Thorac Oncol. 2016;11: S262-3.

17. Alexandraki I, Mooradian AD. Barriers related to mammography use for breast cancer screening among minority women. J Natl Med Assoc. 2010;102:206-18.

18. Silvestri GA, Nietert PJ, Zoller J, Carter C, Bradford D. Attitudes towards screening for lung cancer among smokers and their non-smoking counterparts. Thorax. 2007;62:126-30.
19. Pinsky PF, Gierada DS, Black W, Munden R, Nath H, Aberle D, et al. Performance of lung-RADS in the national lung screening trial: a retrospective assessment. Ann Intern Med. 2015;162:485-91.

20. Kinsinger LS, Anderson C, Kim J, Larson M, Chan SH, King HA, et al. Implementation of lung cancer screening in the Veterans Health Administration. JAMA Intern Med. 2017;177:399-406.

21. Wilson DO, Weissfeld JL, Fuhrman CR, Fisher SN, Balogh P, Landreneau RJ, et al. The Pittsburgh lung screening study (PLuSS): outcomes within 3 years of a first computed tomography scan. Am J Respir Crit Care Med. 2008;178: 956-61.

22. Tanner NT, Gebregziabher M, Hughes Halbert C, Payne E, Egede LE Silvestri GA. Racial differences in outcomes within the national lung screening trial: implications for widespread implementation. Am J Respir Crit Care Med. 2015;192:200-8.

23. Crestanello JA, Allen MS, Jett JR, Cassivi SD, Nichols FC III, Swensen SJ, et al Thoracic surgical operations in patients enrolled in a computed tomography screening trial. J Thorac Cardiovasc Surg. 2004;128:254-9.

24. Flores R, Bauer T, Aye R, Andaz S, Kohman L, Sheppard B, et al. Balancing curability and unnecessary surgery in the context of computed tomography screening for lung cancer. J Thorac Cardiovasc Surg. 2014;147:1619-26.

25. Miller DL, Mayfield WR, Luu TD, Helms GA, Muster AR, Beckler VJ, et al Community-based multidisciplinary computed tomography screening program improves lung cancer survival. Ann Thorac Surg. 2016;101:1864-9.

Key Words: lung cancer screening, safety net hospital, socioeconomic barriers, lung-RADS, follow-up, compliance

\section{Discussion}

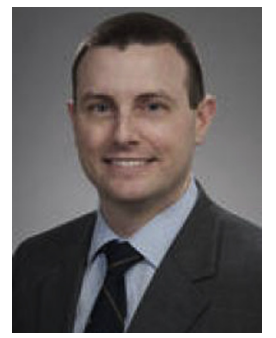

Dr Robert Meguid (Englewood, Colo). Congratulations Dr Litle, to you and your coauthors on the presentation of the manuscript and on successfully performing this presentation, and thanks for sending me the manuscript ahead of time. So, you've retrospectively assessed the outcomes of over 500 patients seen in your lung cancer screening clinic at a safety net hospital. As you pointed out, the population is at high risk of lung cancer, and traditionally has poor access to health care. You demonstrated the successful limitation and your findings are very similar to those in the NLST in the real world setting if you will and other lung cancer screening studies, and so this validates your __. Based on your demonstrated success, what advice do you have to offer other safety net hospitals

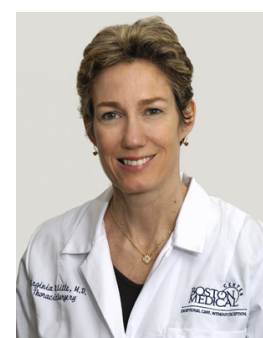

Dr Virginia Litle. I had the programmatic goals outlined there. The steering committee led by a pulmonologist was key and included chest radiologists, pulmonologists, thoracic surgeons, social workers, hospital administrators, and marketing people. IT involvement was also important. I think just having a good committee to organize everything is of course important, as is establishing patient navigators. Money helps too! 
Dr Meguid. As you discussed a little bit, there is a surprisingly large amount of missing key data specifically, about $1 / 4$ of patients who have known active smoking status and then about $20 \%$ of patients that had no status You touched on this, but what are your thoughts about collecting

Dr Litle. I think probably collecting it when they come in for their CT scans is the best place for them when they're going to have the scan, so probably in the Radiology Department.

Dr Meguid. And then you mentioned that some of the patients who underwent lung screening didn't particularly meet the US Task Force guidelines based on . Out of curiosity, did this include any did any of the criteria? Or were they all criteria patients?

Dr Litle. Yes, 10 of the 11 fit the criteria. One didn't. He was 53, but he was a smoker.

Dr Meguid. Yes

Dr Litle. Yes, he was one, he actually had had a history of head and neck cancer, so I think someone decided

Dr Meguid. And a final question. I noted that 2 of your patients had IIIA disease, but neither of those patients made it to surgery is what it sounded like.

Dr Litle. Right, they were poor operative candidates, so they were offered definitive chemoradiation.

Dr Meguid. That's not elucidated in the manuscript, so as a thoracic surgeon, I would encourage you to do that.

Dr Litle. Great, we will add that.

Dr Meguid. Excellent presentation.

Dr Litle. Okay, thanks Rob.

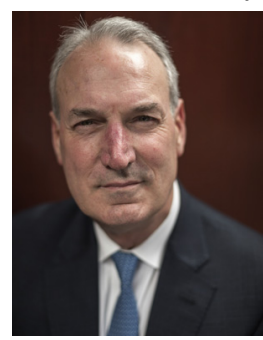

Dr Douglas E. Wood (Seattle, Wash). Dr Litle, that was a great presentation, and congratulations on achieving such incredibly reliable follow-up in a challenging population. I think anyone would be proud of getting 98\% adherence to LungRADS 4 follow-up protocols. I mean, that's almost impossible to achieve. And it's especially important that you've demonstrated that this can be done in a population that has suffered from healthcare disparities, because that certainly is the case with lung cancer patients, as lung cancer disproportionately affects lower socioeconomic groups, minorities, etc. So the fact that you've been able to achieve these results in a safety net hospital is really important information. My question relates to what Dr Meguid was just asking, relating to the number of patients who underwent operations, which is 5 of the 11 patients who ultimately had cancer. Recognizing that a couple of them had higher-stage cancers than we would normally expect with a screening program, there's another aspect at play here: whether patients are being referred for screening who should not be referred for screening, because they're not candidates for what we would consider the standard of care for an early-stage lung cancer. You had a patient with limitedstage small cell cancer who also might have been a candidate for surgery, so is there a way that we can better educate our primary care providers or pulmonologists so as to avoid high-comorbidity patients who might not benefit from screening from getting enrolled into a screening population?

Dr Litle. Thank you very much for your feedback and for your question. You make a very good point. We need to determine...well, educate our referring doctors or those who are placing the order for the screening CT scan, and perhaps we need another hard stop at again, the Radiology Department. I think that might be the best place. I welcome any suggestions from those who have programs, because it's hard for us to reach all the providers out in the community. Perhaps additional integration into EPIC will help us with data compliance.

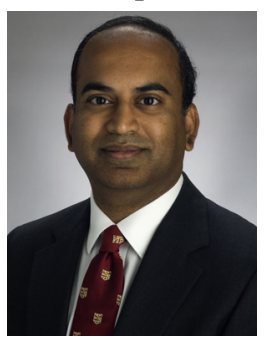

Dr Nirmal Veeramachaneni (Lawrence, Kan). Thank you for a terrific presentation on something that can be logistically very challenging to set up. I just had some questions on some of the finances and the logistics of what you have accomplished. CMS, unlike for other screening programs for breast or colon, has set a lot of logistic hurdles on how to implement lung cancer screening and the requirements for datatracking. So a couple of questions. If a patient has no insurance, how do you handle this? Who does all the face-to-face encounters? When we tried this, it's not economically viable for a pulmonologist to be doing it, so we have a nurse practitioner dealing with the face-to-face encounter, and how do you deal with the database management that's required?

Dr Litle. So, you're saying after they've already been screened and need their follow-up and...is that what you're saying?

Dr Veeramachaneni. No, I mean just to be eligible for screening, there's a mandated face-to-face encounter that CMS has, where you have to talk to them about their risk of lifelong radiation...

Dr Litle. And smoking cessation and all that sort of stuff.

Dr Veeramachaneni. ...etc., how do you handle that?

Dr Litle. Well, I don't think we're handling it very well yet, because again, we're getting folks from the community health centers just ordering the scans after checking off that the counseling was done. That is on our to-do list to figure out how to improve that. Any additional suggestions would be helpful.

Dr Veeramachaneni. So trying to meet CMS hurdles has been one of our biggest challenges, and the way we've done this is nurse practitioner-driven, where I supervise the nurse practitioner, because radiologists aren't comfortable doing 
the screening, and then addressing some of the issues, should the patient even be screened. If we get a patient who comes in a wheelchair and on oxygen, we tell them, "Forget it."

Dr Litle. Right, I think right now people are just placing the order and patients are getting the scan, then they're coming to our Lung Nodule Clinic or Thoracic Clinic, so we then counsel them on the results.

Questioner. I just have a brief question for you. Elaborate a little bit about recruitment again. You intimate that a lot of the patients come your way through the primary care providers and the clinics, but how much selfreferral is there? How much advertising do you do in this patient population, how effective? So, what are your techniques?
Dr Litle. We have advertising and brochures that we've sent to the community health centers and to primary care physicians within our hospital. I don't know the proportion of self-referrals. I suspect that it is low, but I don't know the actual number. Pulmonologists order them, some thoracic surgeons have ordered them although I'm not sure who, if I didn't do it... and the primary care, and the community health center clinicians are ordering it too. We're just continuing to educate those in our referral base about what the criteria are, so that they can make appropriate referrals, and I think maybe the next step is probably have them refer to Radiology (or maybe lung nodule clinic?) to make sure that we're only screening the appropriate population and not wasting money and exposing patients to unnecessary radiation. 